\title{
Design of Yarn Tension System and Jacquard Knitting Machine
}

\author{
Li Zhu \\ School of Mechanical Engineering and Automation \\ Wuhan Textile University \\ Wuhan, China \\ zlcurie@126.com
}

\author{
Xiaoguang $\mathrm{Wu}$ \\ School of Mechanical Engineering and Automation \\ Wuhan Textile University \\ Wuhan, China \\ 2008wuxiaoguang@wtu.edu.cn
}

\begin{abstract}
In view of high fault rates of tension control system in the traditional circular knitting machine, this paper proposes and develops one kind of monolithic integrated circuit yarn tension control system. By using the FTA method to analysis systematically each kind of trouble cause in yarn tension system, and analysis influencing reliable factor of real-time control system, established fault tree the circular knitting machine tension control system. Based on the MCU technology, the sensor network in the program is constituted by ordinary sensor node, the sink node and network coordinator, and responsible for acquiring and transmitting condition information of circular knitting machines. The tension control system has been entered experiment movement with good performance, it is indicated that the this study is benefit to enhance the reliability of yarn tension system.
\end{abstract}

Keywords-Yarn tension; Jacquard Knitting Machine; MCU design; Control System; Tesnsion Parameter

\section{INTRODUCTION}

The traditional circular knitting machine with yarn feeding mechanism to control the feeding amount of negative type, the feeding mode due to yarn supply amount is not the same, leading to the yarn tension fluctuation, high breakage, low production efficiency, low product quality. Using single motor control system, due to the complex mechanical structure, internal and external connection structure is complex, the transmission accuracy can not be guaranteed, the fault occurred frequently, lead to the low reliability of circular knitting machine. As the motor control technology to improve the control precision of the rise, now increasingly use multi motor control. In view of the above situation, decided to use the machine has the advantages of simple structure, multi motor control system has high reliability of motor control system to replace the original single. Multi motor control system using the embedded MCU module control structure, each module with different properties, the influence on the system is also different, so the article using the fault tree analysis of coherent analysis of various fault factors driving mechanism for yarn feeding on circular knitting machine, the fault tree was built.
The use of fault tree (FTA) method can make the various factors that cause the system according to the tree structure part of the overall analysis, determine the cause system failure may combination and probability, so as to find out the weakest link of the system and to develop targeted improvement measures. Finally, based on yarn's control system working condition. We adopt on-line redundancy technology and the other anti-interference technology to improve the reliability of system, to control yarn dynamic tension balance.

\section{DETERMINATION OF FAULT TREE}

The method of fault tree analysis is in the system design process, through a variety of factors causing system faults on possible (including hardware, software, structure, environment, human factors) analysis. Establishing the fault tree should first for systematic analysis of various fault state of the system, the system is the most undesirable failure as the top event, and around the layers of in-depth analysis of the fault state, to find out the influence of all kinds of faults on the fault system, express the internal relations, so as to establish the fault event logic tree.

This system adopted by the speed control of the yarn tension size, AT89S51 MCU is used to control the motor speed, improvement project in the yarn feeding mechanism, through to the fault tree analysis, provides the basis for the reliability design of the system. Fault tree in the fault control caused by yarn tension fluctuation as the top event. In the computer control system, the cause of each sub system can not work normally include two aspects of hardware and software. Therefore, here we only consider the hardware factors and mechanism factors. These interferences which directly action on the system software and hardware could cause system's production breakdown.

Microcomputer control system design according to the module, the module of the system mainly includes power supply control module, motor control module and auxiliary circuit, signal detection and control module, communication module. Any module can make the system failure.

Signal detection module: mainly completes signal acquisition, $\mathrm{AD}$ conversion and liquid crystal display. 
The motor control module: This module mainly by the motor speed to control the tension of yarn size. When tension is greater than the preset value, to control the speed of the motor. Conversely, when tension is less than a preset value, the motor speed reduction control.

Communication Module: communicate with upper computer.

Regulated power supply module: the system used by the DC voltage of AT89S52 power supply voltage of $5 \mathrm{~V}$ and a permanent magnet DC motor power supply voltage of $\pm 48 \mathrm{~V}$ and measuring sensor supply voltage $24 \mathrm{~V}$. CPU supply voltage is directly related to the reliability of the control system hardware, software. The design idea of the regulated power supply is: $24 \mathrm{~V}$ DC voltage as a power supply controller. $5 \mathrm{~V}, \pm 48 \mathrm{~V}, 24 \mathrm{~V}$ is the output of the controller.

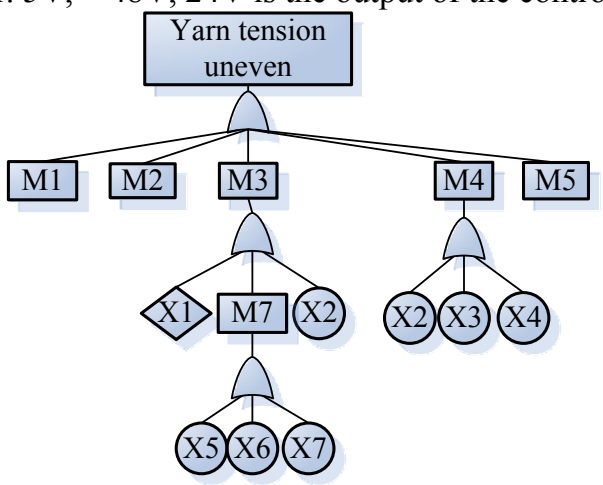

Figure 1. Yarn tension uneven fault tree

As shown in Fig.1, real-time monitoring and control to the yarn tension in this system. belong to the specially design of the hardware and software in the real time inspecting and control, which make the difference to the ordinary system. The coding meaning is shown in table 1 .

TABLE I. CODING MEANING

\begin{tabular}{|c|l|c|l|}
\hline M1 & Power supply fault & X1 & $\begin{array}{l}\text { Control } \\
\text { circuit failure }\end{array}$ \\
\hline M2 & CPU default & X2 & $\begin{array}{l}\text { Electrical componen } \\
\text { f failure }\end{array}$ \\
\hline M3 & $\begin{array}{l}\text { Motor } \\
\text { control module fault }\end{array}$ & X3 & Open circuit fault \\
\hline M4 & Communication fault & X4 & Brush wear \\
\hline M5 & Signal test failure & X5 & $\begin{array}{l}\text { Elastic double beam } \\
\text { failure }\end{array}$ \\
\hline
\end{tabular}

\section{QUALITATIVE AND QUANTITATIVE ANALYSIS} OF FAULT TREE

To monitor the working state of CKM, install vibration sensors adjacent to yarn in the previous location of the feed yarnas to detect needle breaking event, which happens with a vibration, this vibration signals are the acquired fault information.

Fault tree is a cut set represents a probability of failures of the system, which is a failure mode. Since the minimum cut set occurs when the top event must occur. The minimum cut sets on system fault model, the minimum cut set is the meaning of the basic fault it depicts system malfunction state which we must pay attention to, and points out the weak link in the system. For the minimum cut sets method has 2 kinds: ascending method and descending method The According to algorithm, get all the minimal cut sets of $\left\{M_{1}\right\},\left\{M_{4}\right\},\left\{M_{6}\right\},\left\{X_{1}\right\},\left\{X_{2}\right\},\left\{X_{3}\right\},\left\{X_{4}\right\},\left\{X_{5}\right\}$.

A minimal cut sets of the fault tree structure function is set as follows:

$$
\phi(x)=\bigcup_{i=1}^{k} C_{i}
$$

Here, $C_{1}, \quad C_{2}, \ldots, C_{k}(\mathrm{~K}$ is the minimum cut set number) and the probability of basic events $x_{1}, x_{2}, \ldots$, $x_{n}$ is known for $P_{i}$, the effectiveness of probability is the minimum cut sets for:

$$
P\left(C_{j}\right)=P\left(x_{1} \cap x_{2} \cap \cdots \cap x_{n}\right)=\prod_{i=1}^{n} P_{i}
$$

$\mathrm{P}$ event probability of $\mathrm{T}$ is:

$$
P(T)=P\left(\bigcup_{j=1}^{k} C_{j}\right)
$$

And the minimal cut $C_{j}(X)$ sets IS independent, so at this time:

$$
P(T)=1-\prod_{j=1}^{k}\left(1-P_{C_{j}}\right)
$$

Each module in the system for electronic products and components, hardware fault show that the exponential distribution, the failure rate $\lambda$ is constant. The corresponding modules are connected in series to form the independent subsystem also obeys the exponential failure distribution, module fault do not influence each other, independent, rate of each module of the system failure rate and failure. Reliability is the product under stipulated conditions and within the specified time to complete the function, the probability, the system reliability:

$$
R_{s}(t)=P(T)=1-\coprod_{j=1}^{k}\left(1-e^{-\lambda_{X_{i}} t}\right)
$$

Through making a quantitative analysis of fault tree, failure probability of top event can be determined, more important is to analyze the importance of bottom events. The important degree is set on the top event failure probability of the contribution of a component or system, the application in the aspect of system design, fault diagnosis and optimization design. Important is the probability importance degree and the important degree of structure, and the probability of bottom event probability of impact on the top event..

The control chart and find the weakness of single chip computer control system, and puts forward the solutions and measures should be taken in the design of the system

1. It can be seen that, the most obvious influence the reliability of the system is regulated power supply module. Power module is mainly electronic tube, capacitor, diode.

2. Fault of motor control module is larger, and the output tension value. More attention should be paid to. Fault motor control system mainly includes inter turn short circuit, open circuit, brush wear. Because the brush wear the highest failure rate, the main reason is the poor 
working environment, yarn catkins winding of the wear more quickly, thus to improve the electrical controller space clean and seal.

3. Input and output module includes signal detection $\mathrm{m}$ odule and output control signal. As election of conversion speed AD converter, the selection of measuring copper ela stic material tension into double beam to improve the frequ ency and accuracy.

4. With the increase of time of each module, fault rate is increasing, so the need to strengthen the maintenance regularly, discover the problem.

\section{IMPROVED DESIGN}

Based on the minimal cut sets of the fault tree can reach atop event occurrence probability. If can make the probability of events can be decreased, the probability of top event effectively reduce. From above analysis, in order to improve the system reliability, we should improve the reliability of the hardware. In the circuit design should try to control the noise source, noise propagation and coupling, reduce noise absorption. Secondly, select components of high level, and improve the electrical contacts of the clean and seal. The MTTF to $2.0 \mathrm{e}+6 \mathrm{~h}$; on the other hand, improve the control system software reliability. Factors affecting the software reliability: requirement definition error (such as developers and users demand for understanding different); design error; coding errors; interference caused by error (communication error, the program runs away). Therefore, in the design of the system the SCM system boot detection; in the process of adding a periodic output end refresh command; by every $10 \mathrm{~ms}$ repeated sampling of the signal; application of modular design method divides the whole program is divided into several independent small procedures, the inspection and check the convenience.

By improving the design, the yarn tension output balance, the system reliability is greatly improved. In the actual circuit, there are many factors influencing the reliability of each module, and because of the different working environment and each component quality level and the operating frequency is not the same, so the ideal condition of the calculated data with the actual data has certain discrepancy. But through the analysis method of fault tree, still can find out the various factors affecting the reliability of the system.

Testing and research on the mechanical properties of yarn in dynamic conditions have more practical significance. CCT yarn Performance Tester, made by American Lawson-Hemp Hill provides the possibility for yarn dynamic test. As the yarn is in motion state, each part of the yarn samples can be detected in the test process when using the CCT yarn Performance Tester to test its dynamic tension, the obtained yarn-tension value, therefore, is worth more effectively and meaningfully. Furthermore, the yarn being in different tension and operating speed plays a very important role in maintain the continuity of yarn.

\section{DESIGN OF SENSOR}

\section{A. Parameter calculation}

Dynamic tension is usually little in textile industry, specially in knit production, so the sensor should have high sensitivity and resolution. In addition, sensor must have fast response speed in order to reflect the pulse's variation of tension, e.i. elastic component should have higher inherent frequency. It is contradictive and influencing between the sensitivity and the inherent frequency, so the inherent frequency is more considered, we have to increase device's magnified ability to meet the requirement of sensitivity on the premise of proving dynamic response.

Cotton yarns are often used in experiment, the relative formula and linear density as below:

$$
N_{e}=\frac{\left(100+W_{k}\right) / 100}{\left(100+W_{e}\right) / 100} \cdot \frac{590.5}{N_{t}}=\frac{C}{N_{t}}
$$

$W_{k}$ is the commercial moisture regain, $W_{e}$ is the British regain(regain is the physical unit which measures the humidity degree of the yarn), which the pure cotton fiber is $8.5 \%$ and $9.89 \%$. NT is the linear density (which is the term usually used to measure the fineness of the yarn), and the unit is tex. Through counting accordingly, the linear density of the cotton is 18.2 tex while the Ne is $30 \mathrm{~s}$, and the leaner density is 14.6 tex while $\mathrm{Ne}$ is $40 \mathrm{~s}$.

The calculation of testing inherent frequency of roll: we elect duralumin as the materials of the cantilever beam when we use the tension machine sensor which type is LC 1122. In order to realize the requirement of the pitch and equipment, we set the $\mathrm{b}$ is $10 \mathrm{~mm}, 1$ is $45 \mathrm{~mm}$, the thickness of the material $\mathrm{h}$ is $1.8 \mathrm{~mm}$, the elastic modulus $\mathrm{E}$ is $0.72 \times 105 \mathrm{~kg} / \mathrm{mm}^{2}$, the specific density $\rho$ is $2.8 \mathrm{~g} / \mathrm{cm}$.

\section{B. Sensors}

For the monitoring end, the device should be installed appropriate sensors in accordance with the actual needs to obtain necessary parameters. For example, the calculation of the cloth output can be realized by setting detection sensors in the needle cylinder of the circular knitting machine to acquiring the number of revolutions. According to the conversion formula cloth production:

$$
\mathrm{L}=\mathrm{C} \times \mathrm{K} \times(1-\mathrm{R})
$$

Where: $\mathrm{L}$ is actual fabric production; $\mathrm{C}$ is the number of cylinder revolutions; $\mathrm{K}$ is the cloth length coefficient; and $\mathrm{R}$ is the material shrinkage rate. shrinkage rate is the inherent characteristics of knitted material, length coefficient is on behalf of how much cloth the machine weave per revolution, for the same piece of equipment the two can be seen as known parameters, then induce the data acquired by the sensors into the formula and calculate the weaving cloth output length L.

From the above approximate calculation, this kind of elastic component construction can meet the requirement of the tension test, which the variable frequency of the tension can be less then $70 \mathrm{~Hz}$. Alternatively, we can design the more suitable elastic components according to the actual product. 


\section{CONCLUSION}

Tension variation plays an important role in actual spinning process. tension meter is an instrument which can measure tension through a mechanical recorder.

The detection system of the yarn tension based on MCU platform, which can be applied in the actual product, can test and describe definitely the condition of the yarn dynamic test and provide the principle for evaluating equality of the yarn tension machine's property. By comparison and analysis with the parameters of the advanced yarn tension machine made in oversea, it can make some suggestions for the manufactory of the yarn about the product designation, produce and equipment.

\section{ACKNOWLEDGMENT}

This paper is supported by National Natural Science Fund(51175384) and Natural Science Fund of Hubei(2011CDB216). The author thanks all the reviewers of manuscript.

\section{REFERENCES}

[1] Jin-long Gong, Shu-min Zhao. Design of a utility tension controller, Mechanical \& Electrical Engineering Magazine, 23(6), Sept. 2009, P 50-52.
[2] WANG Yan, ZHANG Guo-shan. A Design of Wireless Sensor Networks Based on ZigBee. Microcomputer Information, 2008, 24(10): 158-160.

[3] Fang Yao, Shuang-Hua Yang, Bokai Xia,et al. Home Automation: System Design and Implementation[J]. Measurement and Control, 2008, 41(10): 310-314.

[4] Zai-hai Yu, Wen-ying Wu, Ruan-qi Chen. Measurements of Yarn Tension in Textile Process, Sensorworld, Jan.2009

[5] Wang Y, Teague T L, west H, eta1. Algorithm aided fault tree synthesis for control loops[J]. Journal of Loss Prevention in the process Industries, 2009 (16):427-441

[6] HE Yong, YANG Yan-zhu, WANG Ning. Design for a New Model of Yarn Tension Auto-control System[J]. journal of donghua university(natural science). 2008

[7] Edgar H, Callawy J, Callaway E H. Wireless Sensor Networks: Architectures and Protocols [M]. New York: A uerbach Publications, 2010:260-300.

[8] HUANG Yi, DUAN Jian, DA ILiming, QIN Keli. Control system of magnetically suspended table based on linear quadratic optimization. Modern manufacturing engineering. 2009. No. 8. P108-112.

[9] XIAO Shuaifei, HE Peixiang, LI Qingdong, et al. The repulsive force characteristic of hybrid magnetic levitation system[J]. Science and Technology Innovation Herald, 2010(29) : 71 - 72

[10] Wang Y Q, Jia Y Z, Qia J, et al. load spectra of CNC machine tools. Quality and reliability Engineering International, 2008, 16(3): 229 234 